\title{
Sarcopenia, una patología nueva que impacta a la vejez
}

\author{
William Arbey Gutiérrez Cortés ${ }^{1}$, Fhara Estefanía Martínez Fernández ${ }^{2}$ \\ Laura Camila Olaya Sanmiguel ${ }^{2}$
}

\author{
${ }^{1}$ Médico especialista en Geriatría Clínica, Pontificia Universidad \\ Javeriana; Especialista en Epidemiología, Universidad \\ Surcolombiana; Médico Geriatra, Clínica Mediláser; Docente \\ Catedrático, Universidad Navarra. \\ ${ }^{2}$ Estudiantes VIII semestre de Medicina, Fundación Universitaria \\ Navarra. \\ Fecha de recepción: 30/11/2017 \\ Fecha de aceptación: 31/01/2018
}

\section{Resumen}

$\mathrm{L}$ a sarcopenia es un síndrome frecuente en los ancianos, caracterizado por una pérdida gradual, marcada y generalizada de masa muscular esquelética, relacionada con el envejecimiento, que se evidencia en la disminución de fuerza y agilidad, la cual puede generar discapacidad física que a su vez aumenta el grado de dependencia del paciente, disminuyendo así su calidad de vida y aumentando su mortalidad. El Grupo Europeo de Trabajo sobre la Sarcopenia en Personas de Edad Avanzada (EWGSOP, por sus iniciales en inglés) emplea para el diagnóstico de sarcopenia la presencia de los siguientes criterios: masa muscular disminuida y una función muscular deficiente que es determinada por la fuerza o rendimiento del paciente, este diagnóstico se confirma con los criterios que son: 1. Masa muscular baja, 2. Menor fuerza muscular, 3. Menor rendimiento físico. El manejo actual de la sarcopenia emplea un tratamiento no farmacológico que se centra en el cambio de los estilos de vida como son aumentar la actividad física y mejorar la nutrición y, en el tratamiento farmacológico que tiene como objetivo recuperar la funcionalidad, la movilidad y la masa muscular de los pacientes.

Palabras clave: sarcopenia, anciano, epidemiología, diagnóstico, tratamiento.

\section{SUMMARY}

Sarcopenia is a frequent syndrome in the elderly, characterized by a gradual, marked and generalized loss of skeletal muscle mass related to aging, which is evidenced in the reduction of strength and agility, which can generate physical disability which in turn increase the degree of dependence of the patient thus diminishing their quality of life and increased mortality. The European Working Group on Elderly Sarcopenia EWGSOP uses for the diagnosis of sarcopenia the presence of decreased muscle mass and deficient muscular function that is determined by the strength or performance of the patient, this diagnosis is confirmed with the criteria that they are 1. Low muscle mass, 2. Less muscle strength, 3. Less physical performance. The current management of sarcopenia employs a non-pharmacological treatment that focuses on the change of lifestyles such as increasing physical activity and improving nutrition and pharmacological treatment, which aims to recover the functionality, mobility, muscle mass of the patients.

Key Words: sarcopenia, old man, epidemiology, diagnosis, treatment.

\section{Introducción}

La sarcopenia es un término derivado del griego sarx (carne) y penia (pérdida), el cual fue acuñado por primera vez por Irwin Rosenberg en 1989, para referirse a la pérdida de masa muscular con la edad ${ }^{(1)}$. Desde ese entonces hasta nuestra época, este término ha evolucionado y la patología se ha tenido en cuenta, al relacionar la masa muscular con su función y los cambios que presentan con el pasar de los años. La masa muscular disminuye aproximadamente un 3\%-8\% por década a partir de los 30-35 años y se evidencia un aceleramiento al pasar los 60 años ${ }^{(2)}$.

En la actualidad, se encuentran diversas definiciones sobre sarcopenia. Según el Grupo de Trabajo sobre Sarcopenia en las Personas Mayores (EWGSOP) se define como baja masa muscular en combinación con un bajo rendimiento físico o debilidad en la fuerza muscular ${ }^{(3)}$, a diferencia del Grupo de Trabajo Internacional sobre Sarcopenia (IWGS, por sus iniciales en inglés) que lo define como el índice de masa esquelética (masa relativa apendicular a la altura al cuadrado $)^{(3,4)}$.

Es de gran importancia resaltar que, al referirnos a la sarcopenia, su abordaje deberá ser integral, teniendo en cuenta los factores intrínsecos y extrínsecos que acelerarán la progresión de ésta; además, se encuentra incluida dentro de los síndromes geriátricos, donde se asocia con obesidad, malnutrición, caídas, fragilidad y osteoporosis. Es de vital importancia abordar de manera adecuada la enfermedad y sus asociaciones, ya que las caídas en los adultos mayores son la sexta causa de muerte y representan un impacto económico y social propio de esta edad ${ }^{(2,5)}$. 


\section{Epidemiología}

La sarcopenia es una enfermedad de alta prevalencia en los ancianos, se podría decir que afecta a la mayor parte de esta población y se encuentra ligado al proceso de envejecimiento(6).

A nivel mundial, la prevalencia de la sarcopenia es del $5 \%$ al 13\% en las personas de 60 a 70 años, y en los mayores de 80 años u octogenarios alcanza un porcentaje más alto, llegando incluso hasta un $50 \%{ }^{(7)}$. Según la Organización Mundial de la Salud ${ }^{(8)}$, en al año 2000 existían cerca de 600 millones de personas mayores de 60 años y esta cifra aumentará a 1.200 millones para el año 2025. Estimaciones basadas en la prevalencia de la sarcopenia y la población de la Organización Mundial de la Salud sugieren que la sarcopenia afecta a más de 50 millones de personas en la actualidad y afectará a más de 200 millones de personas en los próximos 40 años ${ }^{(3)}$.

La prevalencia de la sarcopenia en Asia varía, dependiendo de diferentes factores como: edad, sexo, método de medición, entre otros; sin embargo, se evidencia un aumento de la prevalencia en relación con el aumento de la edad, en pacientes de sexo masculino, personas con un bajo índice de masa corporal y que viven en hogares geriátricos ${ }^{(9,10)}$. Según los datos encontrados, existe una mayor prevalencia de sarcopenia cuando ésta se define como una masa muscular en relación con la definición actual que adiciona una fuerza muscular baja o un rendimiento físico pobre ${ }^{(9,10)}$. La prevalencia varía de 6,7 a $56,7 \%$ en hombres y de 0,1 a $33,6 \%$ en mujeres, en adultos mayores según la masa muscular baja ${ }^{(12,13)}$, mientras que la prevalencia según la definición actual es 9,6\% a 22,1\% y $7,7 \%$ a $21,8 \%$, respectivamente ${ }^{(9,10,11)}$. La prevalencia de casos según el estudio de Obesidad Sarcopenia Coreana (KSOS), en el que se utilizó la definición de masa muscular baja en pacientes diabéticos, fue del $15,7 \%$ y se encontró que la diabetes era un factor independiente asociado con la sarcopenia ${ }^{(10,11,12,13,14)}$.

En estudios a gran escala se evidencia la prevalencia de esta enfermedad y su estrecha relación con diferentes comorbilidades. Según el estudio Baumgartner et $\mathrm{al}^{(15)}$, donde se analizan 833 personas mayores (varones y mujeres) seleccionados al azar, de los cuales el 13\% corresponden a la edad de 65 años, un $24 \%$ a los 70 años hasta un $50 \%$ en mayores de 80 años, presentó una mayor prevalencia en hombres por encima de 75 años que en las mujeres. También se demostró que la presencia de sarcopenia se asociaba a un incremento de 3 o 4 veces el riesgo de discapacidad, independientemente de la edad, el sexo, la obesidad, la raza, el estatus socioeconómico y comorbilidades asociadas. Según el estudio de cohorte inglesa, el cual usó la definición de sarcopenia de EWGSOP, encontró una prevalencia de $4,6 \%$ en hombres y 7,9\% en mujeres a los 67 años ${ }^{(16)}$.

Otros estudios similares ${ }^{(17,18)}$, que determinan la prevalencia de la sarcopenia mediante la masa muscular con DEXA (radiometría de absorción dual), fue del 28\% para varones y del 52\% para mujeres mayores de 70 años. Iannuzzi-Sucich et al. ${ }^{(19)}$, hace uso del método diagnóstico DEXA, en su población de $195 \mathrm{mu}$ jeres de 64 a 93 años y de 142 varones entre 64 y 92 años, encontrando una prevalencia general de sarcopenia del $22,6 \%$ en mujeres y del 26,8\% en varones. Estos valores ascendían al 31\% y $45 \%$, respectivamente, para hombres y mujeres mayores de 80 años. Por el contrario, Janssen et $\mathrm{al}^{(20,22)}$, utilizando el índice masa magra/masa total y mediante datos de bioimpedanciometría eléctrica extraídos del NHANES III, encontró una prevalencia de sarcopenia de $7 \%$ y $10 \%$ en hombres y mujeres mayores de 60 años, respectivamente ${ }^{(20,22)}$.

En Colombia, el último censo realizado entre mayo 22 del 2005 y mayo 22 del 2006 reportó un total de 42’090.500 de habitantes, de los cuales 2'617.240 son mayores de 65 años, $54,6 \%$ son mujeres y $45,4 \%$ son hombres; con una proporción de $6,6 \%$ de adultos mayores entre 60 y 74 años, y 2,4\% mayores de 75 años $^{(21)}$. Sin embargo, estos son datos relacionados con la población y no con la patología. Actualmente no se cuenta con datos estadísticos reportados en el Ministerio de Salud acerca del comportamiento, prevalencia e incidencia de la sarcopenia a nivel departamental y nacional.

\section{Etiopatología}

Existe una asociación entre la reducción del músculo esquelético con el aumento de la edad, factores genéticos y factores ambientales que contribuyen al desarrollo de la sarcopenia (figura 1).

\section{Músculo esquelético}

La composición del músculo esquelético se lleva a cabo por:

Fibras musculares: Son dependientes de la "función de la actividad de la ATPasa de las isoformas de las cadenas pesadas de miosina"(2).

- Fibras musculares tipo I (fibras rojas): se identifican por generar fuerzas de larga duración, por su metabolismo aerobio, el acúmulo de mitocondrias y su amplia red capilar y mioglobina. Su principal función es la contracción lenta, que genera un aumento en la resistencia y fatiga muscular ${ }^{(23,24,28)}$.

- Fibras musculares tipo II (fibras blancas): su principal característica es la capacidad de generar contracción rápida y su elevada potencia glucolítica. A su vez, estas fibras se dividen en fibras tipo II A y fibras tipo II B, cuya diferencia es el aumento en la resistencia generada por las fibras tipo II A y su elevada capacidad oxidativa, en relación con el tipo $\mathrm{B}^{(23,24,28)}$.

Esta composición estructural de las fibras musculares es afectada por diversos mecanismos que están relacionados con el aumento de la edad. Las fibras tipo II generalmente tienden a disminuir progresivamente con los procesos de envejecimiento, en regulación a este proceso las fibras tipo I no presentan ningún cambio y en compensación aumentan su número, produciendo un desbalance que se evidencia en la disminución de la actividad oxidativa muscular y densidad $\operatorname{capilar}^{(2,23,24,28,29)}$. 


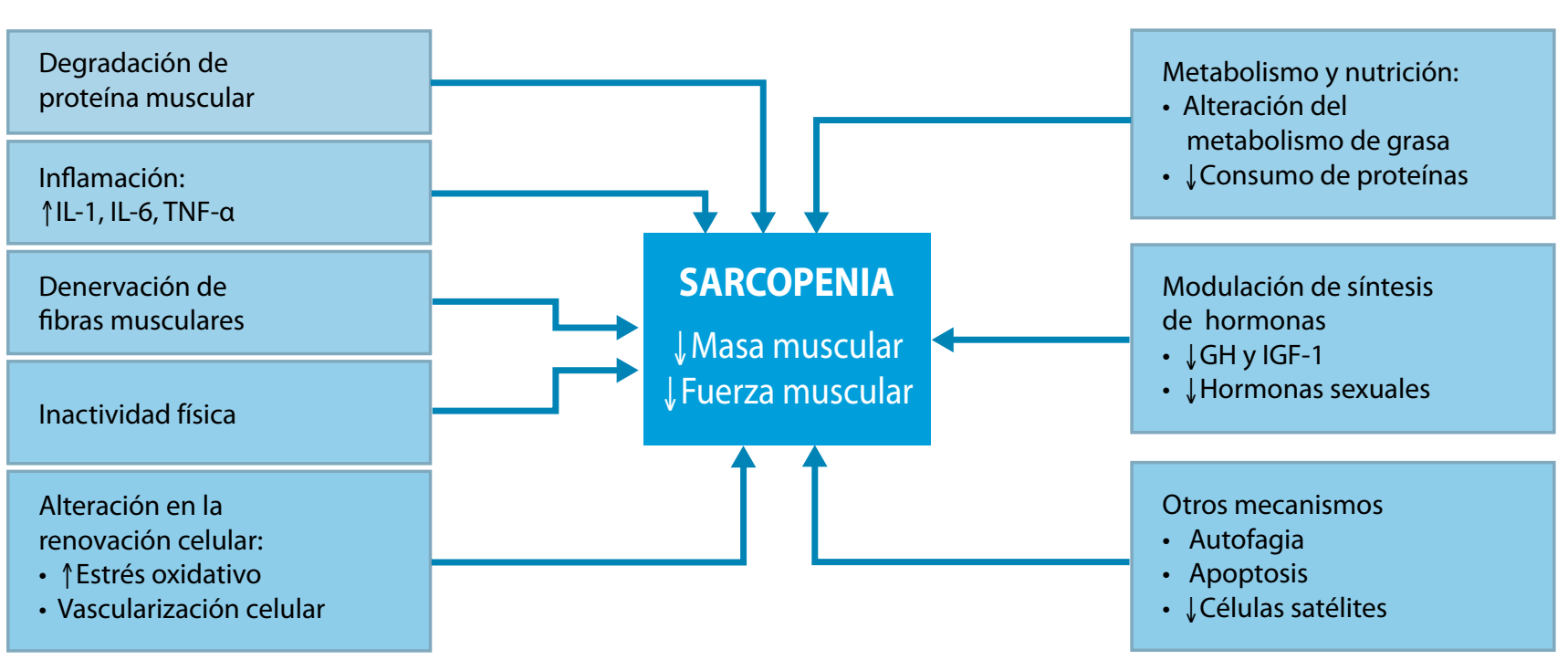

De igual manera, la unidad funcional del músculo "el sarcómero" presenta un proceso degenerativo que se da por medio del remplazo de la fibra muscular propia por tejido fibroso y grasa, lo que conlleva a un acortamiento de las fibras que lo componen y reduce su capacidad de contracción ${ }^{(23)}$. Adicionalmente se evidencia una disminución a nivel central de las unidades motoras alfa del asta anterior de la médula espinal. (figura 2) ${ }^{(2,23,28)}$.

Otro mecanismo por el cual se desarrolla una disminución de las fibras musculares es por medio de factores intrínsecos de los miocitos, que es causado por la acumulación de daños del ADNmt que afecta de manera negativa la síntesis de proteínas, producción de ATP y la tasa metabólica, produciendo senescencia de fibras musculares ${ }^{(24,25)}$.

Durante el proceso de envejecimiento del tejido muscular se evidencia una pérdida de las unidades motoras por medio de la denervación y posterior incorporación en las unidades motoras existentes, produciendo una sobrecarga de trabajo ${ }^{(26,27,28,29)}$.

Pérdida de fuerza muscular: se refleja en la disminución de la contracción de músculo esquelético relacionado con la edad que se evidencia en actividades de la vida diaria como lo son: subir escaleras, ponerse los zapatos, levantarse de una silla, entre otras. Esta disminución se da por igual en ambos sexos y los sitios anatómicos donde se evalúa son los miembros inferiores, siendo éstos los más afectados. Estas se subdividen en isotónicas, isocinética, isométrica ${ }^{(26,27,28)}$.

Las mediciones isocinética e isotónica son utilizadas para medir la fuerza muscular, éstas se hacen en intervalos de tiempo, mientras que la medición isométrica se usa para medir la capacidad de generar fuerza ${ }^{(26)}$.

Figura 2. Resumen de la transformación anatómica del músculo esquelético y el envejecimiento( ${ }^{(2)}$

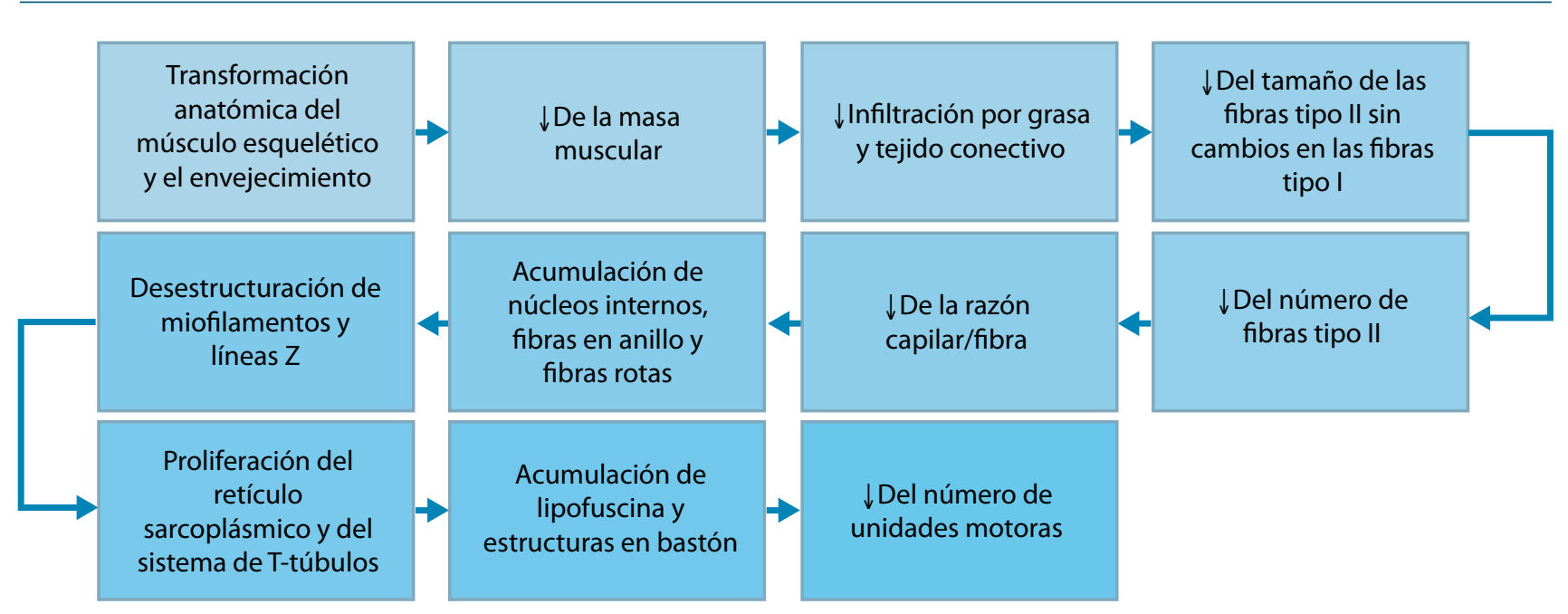


Disminución de la masa muscular esquelética: en el anciano se presenta una disminución de la masa muscular, la cual es universal y progresiva, alcanzando un $3 \%$ por decenio. Dicha disminución aparece desde los 40 años de edad y se acelera hasta en un $15 \%$ después de los 70 años ${ }^{(26,27,29)}$.

\section{Factores genéticos}

El proceso de desarrollo de la sarcopenia puede estar asociado con un "desequilibrio entre la síntesis de proteínas musculares y la degradación"(30); los mecanismos celulares y moleculares que se desarrollan en la sarcopenia, se agrupan en dos vías: la intrínseca y la extrínseca ${ }^{(30)}$.

1. Vía intrínseca: produce cambios en el músculo esquelético ${ }^{(30)}$.

2. Vía extrínseca: cambios en entornos sistémicos ${ }^{(30)}$.

Adicionalmente cuentan con unas vías de señalización que contienen factor de crecimiento similar a la insulina 1 (IGF-1), PtdIns-3-OH cinasa (PI3K), señalización Akt que es la responsable de la síntesis de proteínas musculares, entre otros ${ }^{(30)}$. Existen dos tipos de regulación. La regulación positiva: folistatina, irisina, proteínas morfogénicas del hueso (BMP), factor neurotrófico derivado del cerebro (BDNF) y la regulación negativa: miostatina, activina A y $\mathrm{B}$, factor de crecimiento transformante $\beta$ (TGF- $\beta$ ), el crecimiento y factor de diferenciación (GDF-15). Cuando estos dos tipos de regulación no tienen un balance adecuado, es decir hay una disminución de los factores positivos y un aumento de los factores negativos, se produce la pérdida de masa muscular y posterior aparición de la sarcopenia ${ }^{(23,30)}$.

La muerte celular programada o apoptosis se caracteriza por tener "mecanismos bioquímicos dependientes de la energía", se considera que es vital para varios procesos que realizan las células que componen al ser humano, como son la renovación celular normal, el adecuado desarrollo y funcionamiento del sistema inmune, la muerte celular inducida por procesos químicos, entre otros. Este proceso que se realiza en las células satélites se relaciona con disminución de la masa muscular y el envejecimiento. La destrucción del factor de transcripción Nrf2 dependiente de redox, activa las vías de apoptosis y así disminuye la regeneración muscular. La atrofia muscular que se desarrolla por la denervación tiene un gran susceptibilidad al sistema proteolítico apoptótico mitocondrial, el cual incluye proteosomas y lisosomas que están relacionados con la destrucción de células musculares ${ }^{(30)}$.

En la sarcopenia, la autofagia, que es un proceso por el cual se produce una autodestrucción en la que la célula elimina lo que considera que no es necesario con el fin de generar supervivencia, se asocia con la actividad de ubiquitina -proteasoma y el gen Atg7, que se evidencian en esta patología ${ }^{(30)}$.

La pérdida de masa muscular en la edad avanzada contribuye a la pérdida de fuerza muscular, siendo ésta más significativa en la sarcopenia. Sus células madres (las células satélites) pierden su mayor capacidad de regeneración con el envejecimiento, cambiando su estado de quietud a un estado reversible de senescencia ${ }^{(30)}$.

\section{Factores ambientales}

- Nutrición: según la Organización Mundial de la Salud (OMS), "nutrición es la ingesta de alimentos en relación con las necesidades dietéticas"(31). La disminución de la ingesta de alimentos se relaciona con la edad avanzada, este fenómeno se conoce como anorexia del envejecimiento, la cual se relaciona con una disminución progresiva de la actividad del óxido nítrico y los niveles del neuropéptido (NPY). se evidencia en la disminución del consumo de proteínas en la dieta por diversos factores como son: pérdida de apetito, enlentecimiento del vaciamiento gástrico, niveles elevados de colecistocinina, enfermedades asociadas (cáncer, depresión, demencia), entorno familiar y social, entre otros. La anorexia está relacionada con el desgaste muscular, que puede llegar a generar un estado caquéxico y producir un deterioro funcional progresivo ${ }^{(23,32,33,35,37)}$.

- Disminución de la actividad física: la inmovilidad puede desarrollar cambios fisiopatológicos que pueden conducir a la pérdida de masa muscular, a su vez la pérdida de músculo con la edad, conocida como resistencia anabólica, puede llevar a la inmovilidad ${ }^{(37)}$. Se ha identificado que los hombres y las mujeres en edades avanzadas tienen una menor actividad física, y una menor cantidad de masa muscular esquelética que puede provocar un aumento en la prevalencia de discapacidad ${ }^{(35)}$.

\section{Modulación de la síntesis de hormonas}

- Testosterona: el envejecimiento causa en las células diana una pérdida de su sensibilidad que se evidencia en la relación músculo-testosterona, los niveles de testosterona libre disminuyen aproximadamente un 3\% por año entre las edades de 73 y 94(29) con el aumento de la edad estos procesos disminuyen al desarrollo de sarcopenia ${ }^{(29)}$.

- Hormona de crecimiento (GH): Tiene gran importancia en el estudio de la sarcopenia, debido a que se conoce que su disminución se relaciona con la génesis de ésta ${ }^{(29)}$.

- Categorías: La sarcopenia se puede categorizar en primaria y secundaria, dependiendo de los factores que la desencadenan $^{(3,38)}$ (tabla 1).

Tabla 1. Categorías de sarcopenia ${ }^{(3,38)}$

\section{Sarcopenia primaria $\quad$ Sarcopenia secundaria}

\begin{tabular}{l|l} 
Envejecimiento & $\begin{array}{l}\text { Actividad física: reposo en cama, } \\
\text { gravedad cero, desuso }\end{array}$ \\
\hline $\begin{array}{l}\text { Reducción de las } \\
\text { hormonas sexuales }\end{array}$ & $\begin{array}{l}\text { Enfermedades concomitantes } \\
\text { (corazón, hígado, riñón, pulmón, } \\
\text { cerebro, inflamatorio, endocrino) }\end{array}$ \\
\hline Apoptosis & $\begin{array}{l}\text { Nutrición (inadecuada ingesta } \\
\text { dietética de proteínas) }\end{array}$ \\
\hline Disfunción mitocondrial & \\
\hline
\end{tabular}

Revista Colombiana de Endocrinología, Diabetes y Metabolismo 


\section{Estadificación}

Según EWGSOP, la sarcopenia se estadifica en tres categorías: presarcopenia, sarcopenia y sarcopenia grave ${ }^{(3)}$.

1. Presarcopenia: disminución de la masa muscular, sin cambios en la fuerza muscular ${ }^{(3)}$.

2. Sarcopenia: disminución de la masa muscular y disminución de fuerza muscular ${ }^{(3)}$.

3. Sarcopenia grave: se considera cuando se cumplen los tres criterios que definen la sarcopenia, que son ${ }^{(3)}$ :

- Disminución de masa muscular

- Disminución de fuerza muscular

- Disminución de rendimiento físico

\section{Diagnósticos diferenciales}

Se deben tener en cuenta algunos síndromes geriátricos adicionales como por ejemplo, la fragilidad; entendiendo el síndrome geriátrico como "la conjunción de una serie de enfermedades que alcanzan una enorme prevalencia en el anciano y que son frecuente origen de incapacidad funcional o social".

Fragilidad: Síndrome geriátrico que hace referencia a la disminución de respuesta de diferentes sistemas fisiológicos a estímulos externos nocivos relacionada con la edad y que cursa con pobres desenlaces clínicos y funcionales; su diagnóstico clínico se realiza teniendo en cuenta diferentes criterios, pero uno de los más usados en el ámbito clínico e investigativo es el propuesto por la doctora Linda Fried (fenotipo de fragilidad) el cual enmarca cinco criterios: autopercepción de debilidad y fatiga, disminución de la velocidad de la marcha, disminución de la fuerza de prensión, disminución de la actividad física y pérdida de peso involuntario; teniendo relación directa con la sarcopenia, ya que las dos repercuten en el estado físico y funcional del paciente ${ }^{(3,23)}$.

Osteoporosis: Se define por la disminución de la densidad mineral ósea y deterioro de la microarquitectura de dicho tejido, con aumento de fracturas por fragilidad en el anciano $^{(23,39)}$.

Caquexia: Síndrome metabólico complejo asociado a enfermedades crónicas que hace referencia a la pérdida de peso y masa muscular con o sin pérdida de masa grasa ${ }^{(23,37)}$. Se asocia con anorexia, resistencia a la insulina, inflamación, aumento de la degradación de proteínas musculares; en niños se relaciona con disminución en el crecimiento, su asociación con la sarcopenia se da ya que ésta es uno de los criterios que define caquexia ${ }^{(3,23,37)}$.

Obesidad sarcopénica: Se define por la pérdida de masa magra y el aumento de masa grasa asociado a una enfermedad crónica. La masa corporal es independiente de la disminución de masa muscular y fuerza relacionada con el envejecimiento $^{(3,32,33,36,40)}$.

\section{Diagnóstico}

Según EWGSOP, el diagnóstico de sarcopenia se realiza por medio de los criterios incluidos en la figura $3^{(3)}$.

1. Baja masa muscular

2. Disminución de la fuerza muscular

3. Disminución del rendimiento físico

Estos son evaluados por medio de técnicas de medición de masa, fuerza y desempeño muscular (figura 4).

\section{Evaluación de masa muscular}

Técnicas de imagen corporal: existen varias opciones disponibles para calcular masa grasa y masa muscular ${ }^{(3,23,28,38,41)}$ :

- Tomografía computarizada (TC): evalúa área media muscular del muslo ${ }^{(3,23,28)}$.

- Resonancia magnética (RM): evalúa área media muscular del muslo ${ }^{(3,23)}$.

- Absorciometría dual de Rayos X (DXA): es alternativo en la práctica clínica para diferenciar los tejidos adiposo, mineral óseo y magro ${ }^{(3,23,28,38,41)}$.

- Análisis de bioimpedancia: es usado para calcular el volumen de masa corporal grasa y magra ${ }^{(3,23,28,38,41)}$.

- Excreción de creatinina urinaria: estima la masa ma$\operatorname{gra}^{(3,23,28)}$.

- Medidas antropométricas: medición del diámetro del brazo y pliegues cutáneos. Un diámetro $<31 \mathrm{~cm}$ en la pantorrilla se relaciona con discapacidad. Sin embargo, en la actualidad no se cuenta con parámetros específicos para diagnosticar sarcopenia en el anciano, por tal motivo no es un método diagnóstico de elección ${ }^{(3,23,28,38,41)}$.

Figura 3. Diagnóstico de sarcopenia ${ }^{(3)}$

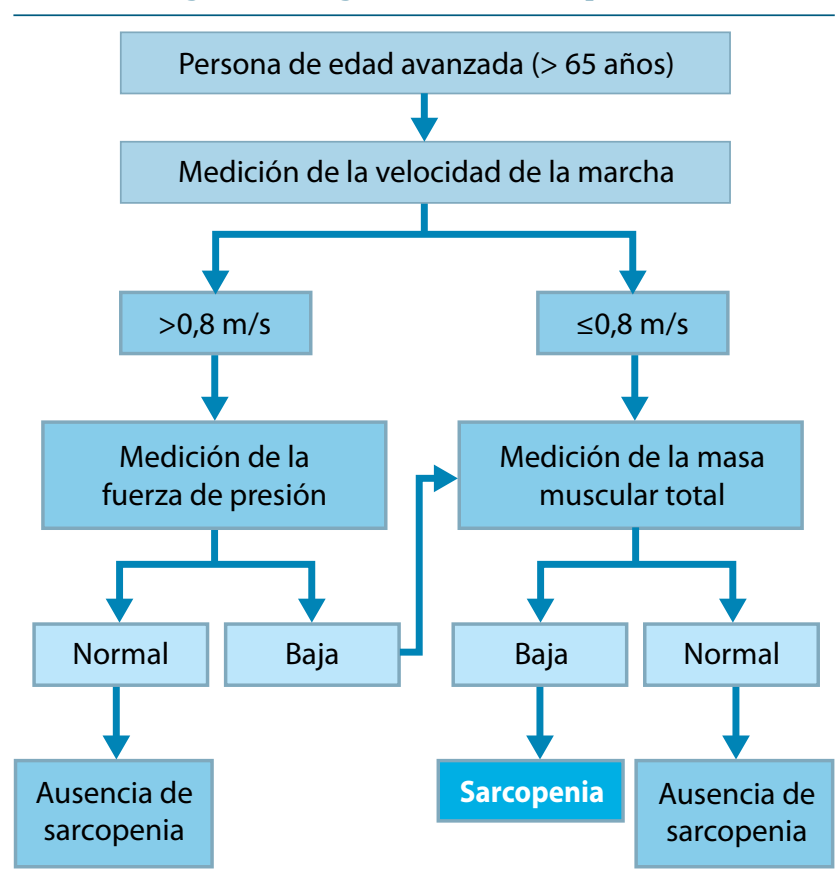


Figura 4. Técnicas de medición de la masa, fuerza y función muscular en investigación y en la práctica clínica ${ }^{(3)}$
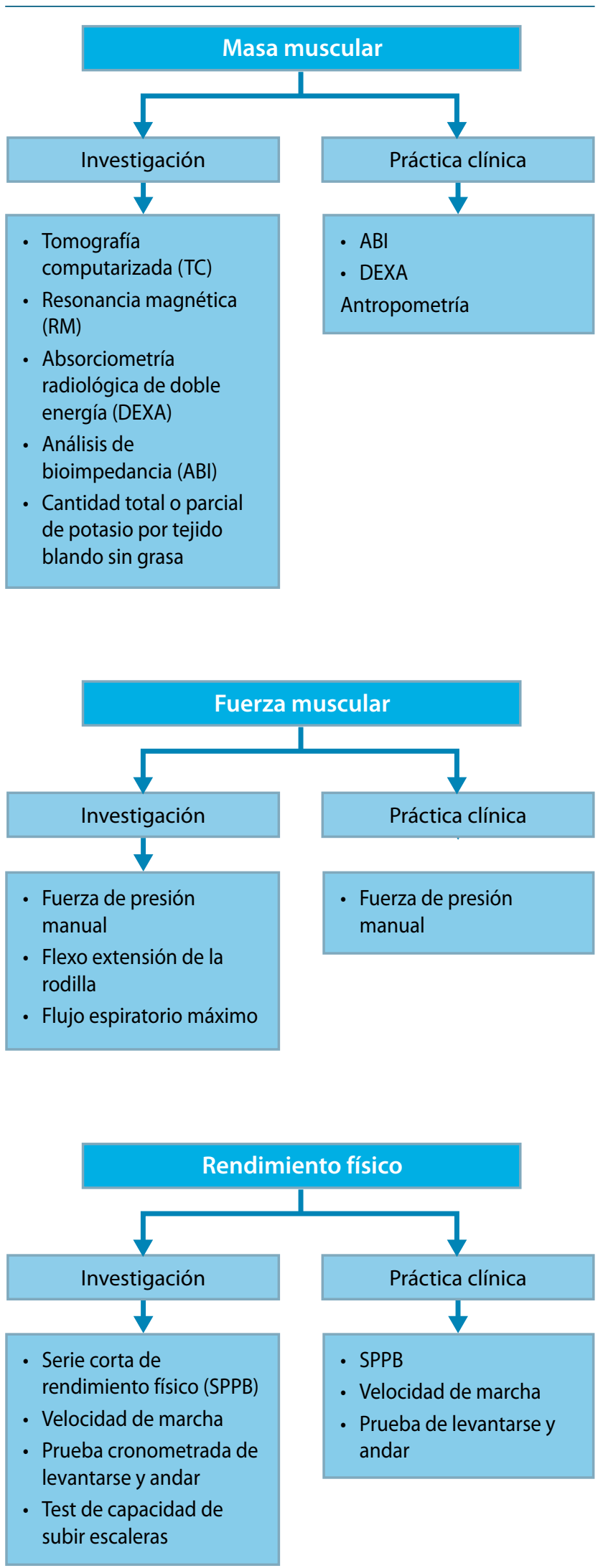

\section{Fuerza muscular}

Fuerza de prensión: es el método más común para medir la fuerza muscular, se realiza con un dinamómetro que evalúa dicha fuerza en la mano dominante de los pacientes, las dos técnicas más frecuentemente utilizadas son las de Lauretani y el sistema propuesto por Fried et al., cuyo punto de corte está determinado por el género (para hombres, $30 \mathrm{~kg}$; para mujeres, $20 \mathrm{~kg}$ ( $^{(28)}$.

- Isométrica: es una técnica manual, donde la longitud de la fibra es constante durante la presencia de una fuerza mayor a la que el músculo puede resistir. Cuando se encuentra disminuida se relaciona con inmovilidad(26).

- Isotónica: donde se cambia la longitud de las fibras musculares contra resistencia de una manera constante ${ }^{(26)}$.

- Isocinética: se produce por un acortamiento o alargamiento de las fibras musculares a una velocidad fija ${ }^{(26)}$.

La medición isocinética e isotónica son utilizadas para medir la fuerza muscular, éstas se hacen en intervalos de tiempo, mientras que la medición isométrica se usa para medir la capacidad de generar fuerza ${ }^{(26)}$.

\section{Resistencia física}

Serie corta de rendimiento físico SPPB: evalúa, marcha, fuerza, equilibrio y resistencia ${ }^{(3,23,28,38,41)}$.

Prueba de levantarse y andar TGUG: se utiliza para medir el tiempo que tarda una persona en realizar una serie de ejercicios. En el adulto mayor sirve como herramienta para evaluar el rendimiento ${ }^{(3,23,28,38,41)}$.

Test de capacidad de subir escaleras: se usa con el fin de medir el rendimiento del paciente $\mathrm{e}^{(3,23,28,38,41)}$.

En la práctica clínica se implementa el cuestionario SARC-F, el cual permite a partir de un breve cuestionario de 5 ítems la detención de sarcopenia en el ámbito ambulatorio, las letras condicionan las siguientes variables: S: lentitud en la marcha, A: apoyo en transferencias, R: levantarse de una silla, C: subir escaleras y por último F: caídas; dicha herramienta cuenta con una especificidad del $94 \%$ al $99 \%$ y una sensibilidad del 10\%.En una cohorte de participantes de la Encuesta Nacional de Salud y Nutrición (NHANES), los puntajes del SARC-F $\geq 4$ se asociaron con una velocidad de la marcha más lenta, menor fuerza y una mayor probabilidad de hospitalización dentro del año de la respuesta de la prueba ${ }^{(28,38,41)}$.

\section{Tratamiento}

El abordaje terapéutico de esta patología se basa en los pilares nutricionales, hormonales, intervencionismo de citocinas en el sistema inmunitario y el ejercicio de resistencia para tratar de reducir la progresión y sus comorbilidades.

\section{Tratamiento nutricional}

La función del aporte proteínico en la dieta de los adultos mayores cobra vital importancia, estudios como el de Volpi et 
$\mathrm{al}^{(42)}$ evidencian que la utilización de aminoácidos medido en biopsias musculares en el muslo demuestra que su incremento aumenta el anabolismo proteínico muscular; lo cual significa que la biodisponibilidad proteínica es importante para el mantenimiento de la masa muscular.

Estudios refieren la importancia de la suplementación dietaria con algunos aminoácidos específicos como: la glutamina, leucina y otros aminoácidos ramificados, entre esos un metabolito de la leucina "beta-hidroxi-beta-metilbutirato" $(2,43,44)$.

En adultos mayores con la utilización de beta-hidroxi-betametilbutirato se ha demostrado aumento de la masa muscular libre de grasa e incremento en la fuerza muscular en combinación con ejercicio, aunque no en todos los grupos musculares analizados $^{(45)}$. Por el contrario, en adultos jóvenes fue más favorable la administración de leucina al evidenciarse un incremento de la masa libre de grasa en conjunto con ejercicio ${ }^{(2)}$.

Los aminoácidos con los cuales se obtienen resultados sobre la capacidad para estimular el anabolismo proteínico muscular son los aminoácidos esenciales; se demostró que utilizando suplementación con $18 \mathrm{~g}$ de una combinación de 10 aminoácidos esenciales, a diferencia de la adición de $22 \mathrm{~g}$ de aminoácidos no esenciales, no produjo ningún efecto adicional en la síntesis proteínica ${ }^{(46)}$; hay que resaltar que ha tenido mejores resultados la utilización de aminoácidos esenciales en utilización concomitante con el ejercicio.

En la población geriátrica se recomienda la ingesta proteínica de 1 a 1,2 g/kg/día ${ }^{(47)}$ o 25 a 30 g/día de proteínas de alto valor biológico ${ }^{(48)}$. Adicionalmente, ensayos clínicos aleatorizados demostraron que la utilización de 25-hidroxivitamina D ayuda a la reducción del riesgo de sarcopenia asociado a la prevención de fracturas ${ }^{(49)}$.

Los criterios para la suplementación de vitamina $\mathrm{D}$ y la dosis se basan principalmente en el estudio Bischoff et al, que demuestra que la suplementación con 800 UI de vitamina D por día resultó beneficiosa en la reducción en el riesgo de caídas $^{(50)}$. El estudio Pfeifer et al. demostró que la suplementación con 1000 mg de calcio más 800 UI de vitamina D por día durante un periodo de 12 meses se asoció con una significativa reducción (39\%) en la exclusión de riesgos a los 20 meses en personas mayores ${ }^{(51)}$.

La Sociedad Americana de Geriatría y la Fundación Nacional de Osteoporosis recomiendan la administración de suplementos de vitamina D, al menos 1.000 y 800 a 1.000 UI por día, respectivamente, así como suplementos de calcio a adultos mayores de 65 años para reducir el riesgo de fracturas y

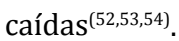

\section{Tratamiento hormonal}

Fisiológicamente, el cuerpo humano después de cierta edad se ve afectado por la inadecuada suplencia de hormonas sexuales que son necesarias para mantener la masa muscular y la fuerza ${ }^{(55,56)}$.
Ensayos clínicos proporcionan evidencia de que el tratamiento con testosterona aumenta la masa muscular esquelética y también la fuerza muscular en cierto grado ${ }^{(57)}$. Por lo general, esta clase de tratamiento se prescribe a los adultos mayores con hipogonadismo, teniendo como prevalencia un $20 \%$ en varones mayores de 60 años, cifra que asciende a un $50 \%$ en varones mayores de 80 años ${ }^{(58)}$.

Este tratamiento genera cierta duda acerca de la seguridad en su aplicación en la población adulta mayor, ya que es más vulnerable a los efectos secundarios como lo son inducir y exacerbar las apneas del sueño, enfermedades cardiovasculares, incrementa la masa eritrocitaria, causa retención de fluidos transitoria y puede producir ginecomastia, además, producir incremento del tamaño de tumores de próstata tanto benignos como malignos, y se discute su efecto en la carcinogénesis de próstata $^{(58)}$.

Respecto a otros tratamientos hormonales, encontramos que la utilización de dehidroepiandrosterona (DHEA) está todavía en investigación como precursor de diversos esteroides sexuales. En el estudio Morales et al. administraron $100 \mathrm{mg}$ de DHEA a personas de 50-65 años, y obtuvieron un incremento en la masa magra y una disminución en la masa grasa, pero solo se evidenció un incremento en la fuerza muscular en hombres, no en mujeres ${ }^{(58)}$.

El tratamiento sustitutivo con la hormona de crecimiento humana $(\mathrm{HGH})$ incrementa la masa muscular y la fuerza del muslo en ambos sexos en adultos jóvenes con hipopituitaris$\mathrm{mo}^{(59,60)}$, pero en ancianos no incrementa la masa muscular ni la fuerza ${ }^{(61,62)}$ y los efectos secundarios, como el síndrome del túnel carpiano, ginecomastia, hiperglucemia, retención de fluidos, artralgias, hipotensión ortostática y elevado índice de abandonos del tratamiento, son mayores ${ }^{(63,64)}$.

\section{Tratamiento en la función inmunitaria}

Se han utilizado diferentes formas para la modulación de la producción de citocinas que se encuentran involucradas en la pérdida de masa libre de grasa en la sarcopenia, como lo son: los ácidos grasos omega 3 se han hecho estudios en animales en donde incrementan la ingesta en caquexias mediadas por citocinas ${ }^{(65)}$, la talidomida va a incrementar la degradación del ARNm de TNF- $\alpha$; en la población adulta mayor no se han encontrado estudios ${ }^{(66)}$, la pentoxifilina va a disminuir la transcripción del ARN mensajero del factor de necrosis tumoral alfa $(\mathrm{TNF}-\alpha)^{(67)}$, en ancianos no hay estudios, solo en pacientes que sufran de caquexia se ven los resultados y, por último, se encuentra el acetato de megestrol que disminuye la producción de interleucina (IL) 1, IL-6 y TNF- $\alpha^{(68)}$, consiguiendo incremento de peso, en las cifras de ingesta, de albúmina, prealbúmina y del recuento de linfocitos en ancianos con 12 semanas de tratamiento ${ }^{(2,69)}$. 


\section{Tratamiento no farmacológico Ejercicio}

La piedra angular del tratamiento para la sarcopenia es el ejercicio de resistencia que ayude al paciente al fortalecimiento y aumento de la masa muscular. El Colegio Americano de Medicina Deportiva y la Asociación Americana de Cardiología recomiendan entrenar con pesas 2 o 3 veces a la semana para lograr aumentos en el tamaño y la fuerza muscular, incluso en personas mayores frágiles ${ }^{(70,71)}$. Los estudios recientes han examinado ejercicios multicomponentes consistentes en fuerza muscular combinada con entrenamiento de equilibrio y marcha en personas nonagenarias frágiles, que muestran un aumento en el área de los músculos transversales, una mayor fuerza muscular y mejora del tiempo y pruebas con una o dos tareas y equilibrio ${ }^{(72)}$.

Los ejercicios que tienen la mejor evidencia son los de alta intensidad ${ }^{(73)}$. El tiempo de tratamiento necesario para observar efectos positivos generalmente es de 10-12 semanas, aunque en algunos estudios se observan efectos positivos con dos semanas de entrenamiento. Ciertos clínicos están en desacuerdo para recomendar este tipo de ejercicios a ancianos, pero hay evidencia de que un entrenamiento adecuado para esta clase de población puede llevarse a cabo con seguridad, incluso en ancianos de mucha edad ${ }^{(74)}$.

Con esta clase de tratamiento los efectos secundarios son escasos, únicamente se limita en pacientes con insuficiencia cardiaca congestiva $^{(2)}$; por el contrario, los efectos beneficiosos del ejercicio pueden ser moderados por la inhibición de la miostatina a través del aumento de la síntesis hepática de folistatina, un inhibidor de la miostatina, y asimismo mediante la estimulación de las respuestas de hormonas anabolizantes, incluidas la hormona del crecimiento y la testosterona ${ }^{(74)}$. Actualmente, la estrategia más completa en el tratamiento de la sarcopenia es la anteriormente mencionada en combinación de suplementos nutricionales.

\section{Conclusiones}

- El envejecimiento es un proceso irreversible al que todo ser humano llega, está ligado con el aumento de patologías que se van desarrollando al pasar los años; ese es el caso de la sarcopenia, cuya aparición es tardía y está incluida dentro del grupo de síndromes geriátricos que afecta a la mayoría de la población mayor de 65 años.

- Este síndrome geriátrico representa un reto para todo el personal de salud que trabaja con adultos de la tercera edad, ya que su prevalencia va en aumento y representa un gran impacto económico en el sistema de salud.

- Aunque las investigaciones recientes asocian diversos factores de riesgo que pueden desencadenar la aparición de sarcopenia, aún no se saben con certeza todos los mecanismos por los cuales se puede desarrollar y su relación con las diferentes patologías que pueden empeorar sus síntomas y que afectan a los adultos mayores.

- Es de vital importancia saber sus características, buscar un diagnóstico oportuno con el fin de prevenir sus complicaciones que afectan la calidad de vida del paciente y de su entorno, convirtiéndose no solo en un problema de salud sino también en problema social.

- Una adecuada nutrición proteínica, el mejorar los hábitos de vida sana e incluir el ejercicio físico con el fin de mantener la masa muscular hasta la vejez, es el mejor método de prevención que se debe buscar en la sarcopenia.

- La valoración geriátrica del paciente con sarcopenia debe ser integral y personalizada, debe incluir al paciente y sus familiares, con el fin de educarlos acerca de esta patología, buscar apoyo para el paciente, si lo requiere, mejorar la adherencia al tratamiento y disminuir el riesgo de mortalidad.

\section{Referencias}

1. Irwin H. Rosenberg, sarcopenia: origins and clinical relevance; clinics geriatrics medicine 27, 2011, páginas 337-339.

2. Burgos Peláez Rosa; sarcopenia en ancianos; unidad de nutrición clínica. Servicio de endocrinología y nutrición. Hospital universitario de bellvitge. l'hospitalet de llobregat. Barcelona. España, endocrinol nutr. 2006; 53(5):335-44.

3. Alfonso J. Cruz-Jentoft et al, sarcopenia: consenso europeo sobre su definición y diagnóstico informe del grupo europeo de trabajo sobre la sarcopenia en personas de edad avanzada; age and ageing, volúmen 39, julio 2010 , páginas 412-423.

4. Kyung Mook Choi; sarcopenia and sarcopenic obesity; endocrinol metab, 2013; volumen 28:86-89.

5. Ian Janssen, W Donald S. Shepard, Peter T. Katzmarzyk, and Ronenn Roubenoff; the healthcare costs of sarcopenia in the united states; american geriatrics society. 2004; volumen 52:80-85.

6. Núñez Flores Dionisio. Prevalencia de la sarcopenia en hospital de día, hospital nacional de geriatría y gerontología, en el segundo semestre de 2012. Revista clínica de la escuela de medicina.2016; volumen 6: no 5.

7. Morley J.E. sarcopenia: diagnosis and treatment. the journal of nutrition, health \& aging.2008; volume 12:452.

8. World Health Organization. ageing and life course. [Internet] 2017 [citado en noviembre de 2017] disponible en url: http://www.who. Int/ageing/ en/.

9. Beaudart Charlotte, Rizzoli Ren, Bruyère Olivier, Reginster Jean-Yves And Biver Emmanuel. sarcopenia: burden and challenges for public health. Archives of public health.2014; volumen 72:45.

10. Bruyere. O, Beaudart. C, Locquet. M, Buckinx. F, Petermans. J, Reginster J-Y. sarcopenia as a public health problema. eurger. 2015; 705:4.

11. Liang-kung chen et al; sarcopenia in asia: consensus report of the asian working group for sarcopenia. jamda.2014; 15: 95-101.

12. Limpawattana Panita, Kotruchin Praew, Pongchaiyakul Chatlert. sarcopenia in asia. elseviere osteoporosis and sarcopenia.2015; volumen 1: 92-97.

13. Em Lau, Hs Lynn. Prevalence of and risk factors for sarcopenia in elderly chinese men and women. j gerontol a biol sci med sci. Febrero 2005; volúmen 60(2):213-6.

14. Tae Nyun Kim et al, prevalence and determinant factors of sarcopenia in patients with type 2 diabetes. Diabetes care. Julio 2010; volume 33: number 7.

15. Baumgartner Rn, Koehler Km, Gallagher D, Romero L, Heymsfield Sb, Ross $\mathrm{Rr}$, Garry Pj, Lindeman Rd. Epidemiology of sarcopenia among the elderly in new mexico. Am j epidemiol. Abril 1998; 147(8):755-63.

16. Harnish P. Patel et al, Prevalence of sarcopenia in community-dwelling older people in the uk using the european working group on sarcopenia in 
older people (ewgsop) definition: findings from the hertfordshire cohort study (hcs). age and ageing ,2013; 42: 378-384.

17. Melton L. Joseph et al, Epidemiology of sarcopenia. jags, 2000; 48:625-630.

18. Dodds Richard Matthew, Roberts Helen Clare, Cooper Cyrus,And Sayer Avan Aihie. The Epidemiology Of Sarcopenia. Journal Of Clinical Densitometry: assessment \& management of músculoskeletal health, 2015; vol: 16.

19. Iannuzzi-Sucich M, Prestwood Km, Kenny Am. Prevalence of sarcopenia and predictors of skeletal muscle mass in healthy, older men and women. $j$ gerontol a biol sci med sci, 2002;57(12):m772-7.

20. Janssen Ian, Phd, Heymsfield Steven B, Ross Robert. Low relative skeletal muscle mass (sarcopenia) in older persons is associated with functional impairment and physical disability. jags, 2002; 50:889-896.

21. DANE Información Estratégica. Censo 2005. [Internet] 2017[citado en noviembre de 2017] disponible en url: http://www.dane.gov.co/index.php/ estadisticas-por-tema/demografia-y-poblacion/censo-general-2005-1/ censo-general-2005.

22. Janssen Ian, The Epidemiology Of Sarcopenia. Clin Geriatr Med ,2011; volumen 27:355-363.

23. Woo Jean. Sarcopenia. Clin Geriatr Med, 2017.

24. Bruno vellas et al, sarcopenia: its asses ment, etiology, pathogenesis, consequences and future perspectives. j nutr health aging. Agosto - septiembre 2008; 12(7): 433-450.

25. J. De D. Beas-Jiménez, G. López-Lluch, I. Sánchez-Martínez, A. Muro-Jiménez, E. Rodríguez-Bies And P. Navas. Sarcopenia: implications of physical exercise in its pathophysiology, prevention And treatment. rev andal med deporte. 2011; 4(4):158-166.

26. T. Lang \& T. Streeper \& P. Cawthon \& K. Baldwin \& D. R. Taaffe \& T. B. Harris. Sarcopenia: etiology, clinical consequences, intervention, and assessment, osteoporos int.2010;21: 543-559.

27. Walston Jeremy D. Sarcopenia In Older Adults. Curr Opin Rheumatol 2012, 24:623-627.

28. Gutiérrez Robledo Luis Miguel, Acosta Sergio Ley. Sarcopenia, diagnóstico diferencial e implicaciones terapéuticas en carlos d'hyver, luis miguel gutiérrez robledo. Geriatría. Tercera edición. México: editorial manual moderno; 2014.721-725.

29. Yu Jie, The etiology and exercise implications of sarcopenia in the elderly. international journal of nursing sciences.2015; 2:199-203

30. Sung Sup Park, Eun-Soo Kwon, Ki-Sun Kwon. Molecular mechanisms and therapeutic interventions in sarcopenia. Osteoporosis and sarcopenia. 2017; 3: 117-122.

31. World Health Organization.Nutrición. [Internet] 2017 [citado en noviembre de 2017] disponible en url: http://www.who.int/topics/nutrition/es/

32. Dasarathy Srinivasan, Merli Manuela. Sarcopenia from mechanism to diagnosis and treatment in liver disease. journal of hepatology .2016; vol. 65:1232-1244.

33. Agustín Aibar-Almazán et al, Sarcopenia and sarcopenic obesity in spanish community-dwelling middleaged and older women: association with balance confidence, fear of falling and fall risk. maturitas. 2018; 107:26-32.

34. World Health Organization. Sobrepeso y obesidad [internet] 2017[citado en noviembre de 2017] URL: http://www.who.int/mediacentre/factsheets/fs311/es/.

35. Timothy J. Doherty. Invited Review: Aging and sarcopenia. j appl physiol.2003; 95: 1717-1727.

36. Androga Lagu, Sharma Deep, Amodu Afolarin And K. Abramowitz Matthew. Sarcopenia, obesity, and mortality in us adults with and without chronic kidney disease. kidney int rep. 2017; 2: 201-211.

37. Sumbul Ali, Jose M. Garcia. Sarcopenia, Cachexia And Aging: Diagnosis, mechanisms and therapeutic options - a mini-review. karger ag, basel .2014:0304-324.

38. Tarantino Umberto et al, Sarcopenia and fragility fractures: molecular and clinical evidence of the bone-muscle interaction. $\mathrm{j}$ bone joint surg am. 2015. 97: 429-37.

39. M.H. Edwards, E.M. Dennison, A. Aihie Sayer, R. Fielding, C. Cooper. Osteoporosis and sarcopenia in older age. Bone. Noviembre 2015; volúmen 80.

40. Batsis John A., Mackenzie Todd A., Lopez-Jimenez Francisco, Bartels Stephen J. Sarcopenia, sarcopenic obesity, and functional impairments in older adults: national health and nutrition examination surveys 1999-2004. Nutrition research .2015 december 2015, pages 1031-1039.

41. Mark H. Edwards, And Bjoern Buehring. Novel approaches to the diagnosis of sarcopenia. journal of clinical densitometry. Octubre 2015; volumen 18: paginas $472-477$.

42. Volpi E Bartels Stephen J Exogenus amino acids stimulate net muscle protein synthesis in the elderly. J. Clinics invest. 1980; 101: 200-7.

43. Peláezburgos R. enfoque terapéutico global de la sarcopenia. nutr. hosp. 2006; 21 (supl. 3): 51-60.

44. Delosreyesad, Bagchi D, Preuss Hg. Overview of resistance training, diet, hormone replacement and nutricional supplements on age-related sarcopenia. A mini-review. Res commun mol pathol pharmacol. 2003; 113:15970.

45. Vukovich Md, Stubbs Nb, Bohlken Rm. Body composition in 70-year old adults responds to dietary b-hydroxy-b-methilbutarate similarly to that of young adults. j nutr. 2001; 131:2049-52.

46. Volpi E, Kobayashi H,Sheffieldmoorem, Wolferr.Essential amino acids are primarily responsible for the amino acid stimulation of muscle protein anabolism in healthy elderly adults. am j clin nutr. 2003; 78:250-8.

47. Rizzoli R. Nutrition And Sarcopenia. Jorunal of clinical Densitometry. 2015; 8: 483-487.

48. Paddon J, Rasmussen,B.B. Dietary Protein recommedations and the prevention of sarcopenia: protein,amino acid metabolism and terapy. Current opinion in clinical nutrition and metanolic care. 2009; 12:86.

49. Dominguez C. Hugo. Nutrición en la sarcopenia. Mundo entrenamiento el deporte bajo evidencia científica. 2017 (11Noviembre). Disponible en: https://mundoentrenamiento.com/nutricion-la-sarcopenia/

50. Bischoff $\mathrm{Ha}$, et al, Effects of vitamin $\mathrm{d}$ and calcium supplementation on falls: a randomized controlled trial. $\mathrm{j}$ bone miner res. 2003; 18: 343-51.

51. Pfeifer M, Begerow B, Minne Hw, Suppan K, Fahrleitner-Pammer A, Dobnig H. Effects of a long-terma vitamin and calcium supplementation on falls and parameters of muscle function in community-dwellling olders individuasl. Osteroporos int 2009; 20(2): 315-322.

52. Geriatrics Society Workgroup On Vitamin D Supplementation For Older Adults. Recommendations abstracted from the American geriatrics society consensus statement on vitamin $d$ for prevention of falls and their consequences. J Am Geriatr Soc 2014; 62(1):147-52.

53. Lappe M.J, Binkley Neil. Vitamin D in sarcopenia/falls. Journal of clinical densitometry. 2015; vol18: 4478-482.

54. La Colla A, Pronsato L, Milanesi L, Vasconsuelo A. 17 beta-estradiol and testosterone in sarcopenia: role of satellite cells. ageing res rev 2015; 24: 166-77.

55. Finkelstein Js, Lee H, Burnett-Bowie Sa, Pallais Jc, Yu Ew, Borges Lf. gonadal steroids and body composition, strength, and sexual function in men. $\mathrm{N}$ Engl J Med 2013; 369:1011-22.

56. Starka L. testosterone treatment of sarcopenia. Vnitr lek 2006; 52:909-11.

57. Park S.S, Kwon E.S, Kwonk.S.Molecular mechanisms and therapeutic interventions in sarcopenia. Osteoporosis and sarcopenia. 2017; vol 3: 117-122.

58. Salomon F,Cuneorc, Hesp R. The Effects of treatment with recombinant human growth hormone on body composition and metabolism in adults with growth hormone deficiency.nenglj med. 1989; 321:1797-803.

59. Jorgensen Jo, Vahl N,Hansentb. Growth hormoneversusplacebo treatment for one year in growth hormone deficient adults: increase in exercise capacity and normalization on body composition. clin endocrinol (oxf). 1996; 45:681.

60. Rudman D et al, Effects of human growth hormonein men over 60 years old. N. Engl Jour Med, 1990; 323:1-6.

61. Papadakis Ma et al, Growth hormone replacement in healthy older men improves body composition but no functional ability. Ann Internal Med. 1996; 124: 708-16.

62. Cohn et al, carpal tunnerl syndrome and gynecomastia during growth hormone treatment of elderly men with low circulating IGF-1 concentrations. Clinics endocrinol. 1993; 39; 417-25.

63. Yarasheski Ke, Zachwieja Jj. Growth hormone therapy for the elderly: the fountain of youth proves toxic. Jama. 1993; 270: 1694 .

64. Tisdale Mj, Dhesi Jk. Inhibition of weight loss by omega3 fatty acids in an experimental cachexia model. Cancer res. 1990; 50:5022-6

65. Yeh Ss, Schuster Mw. Geriatric Cachexia: The Role Of Cytokines. Am j clin nutr. 1999; 70:183-97.

66. Doherty $\mathrm{Gm}$, Jensen Jc, Alexander $\mathrm{Hr}$, et al, pentoxiftline suppression of tumor necrosis factor gene transcription. Surgey. 1991; 110: 192-8.

67. Mantovani G, Maccio A, Esu S, Et al. medroxyprogesterone acetate reduces theinvitro production of cytokines and serotonin envolved in anorexia/ cachexia and emesis by peripheral blood mononuclear cells of cancer patients. Eur j canc. 1997; 33:602-7.

68. Yeh Ss, Wu Sy, Lee Tp, Et Al. Improvement in quality-of-life measures and stimulation of weight gain after treatment with megestrol acetate oral suspension in geriatric cachexia: results of a double-blind placebo-controlled study. j am geriatr soc. 2000; 48:485-92.

69. Nelson Me et al, Physical activity and public health in older adults: recommendation from the American college of sports medicine and the american herat association. Med Sci sports execer 2007; 39: 1435-45.

70. Williams MA, Haskell Wl, Ades Pa,et al. Resitance exercise in individuals with and without cardiovascular disease: 2007 update: Circulatión 2007; 116: 572-84.

71. Cadore El, Casas-Herrero A, Zambom-Ferraresi F, Et Al. Multicomponent exercises including muscle power training enhance muscle mass, power output, and functional outcomes in institutionalized frail nonagenarians. age (dordr). 2014; 36:773-85.

72. Yarasheski Ke. Exercise, aging and muscle protein metabolism.j gerontol biol sci med sci. 2003; 58:918-22.

73. Fiatarone Ma, O'neill Ef, Ryan Nd, Et Al. Exercise training and nutritional supplementation for physical frailty in very elderly people. $\mathrm{N}$ engl $\mathrm{j}$ med. 1994; 330:1769-75.

74. Kraemer Rr, Castracane Vd.Novel Insights Regarding Mechanisms For Treatment Of Sarcopenia. Metabolism 2015; 64:160-2. 